\title{
Saúde mental antes e depois da artroplastia total do joelho*
}

\section{Mind Health Before and After Total Knee Arthroplasty}

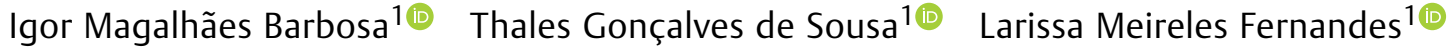

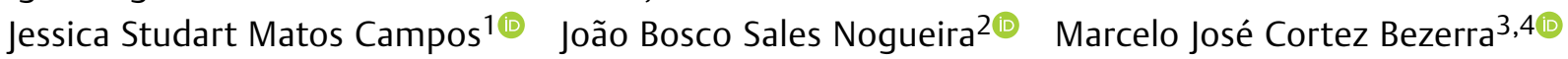

${ }^{1}$ Faculdade de Medicina, Universidade Unichristus, Fortaleza, CE, Brasil

${ }^{2}$ Centro do Joelho, Fortaleza, CE, Brasil

Endereço para correspondência Igor Magalhães Barbosa, MD, Faculdade

${ }^{3}$ Departamento de Ortopedia, Santa Casa da Misericórdia de

Fortaleza, Fortaleza, CE, Brasil

${ }^{4}$ Departamento de Ortopedia, Universidade Unifor, Fortaleza, CE, Brasil de Medicina, Universidade Unichristus, R. Fonseca Lobo, 560, Ap. 1401, Fortaleza, CE, Brasil (e-mail: igormagalhaes1@hotmail.com).

Rev Bras Ortop 2020;55(6):783-786.

\section{Resumo}

Palavras-chave

- artroplastia do joelho

- osteoartrite

- depressão

Abstract
Objetivo Avaliar os resultados pré e pós-operatórios em relação à depressão, dor, limitações funcionais e episódios de queda em pacientes diagnosticados com osteoartrite (OA) grave do joelho submetidos a uma artroplastia total do joelho (ATJ), usando um implante móvel com uma plataforma rotativa e removendo o ligamento cruzado posterior.

Métodos O questionário de Lequesne foi utilizado para avaliar a dor e as limitações funcionais antes e após a ATJ. Além disso, a escala de depressão geriátrica (EDG) também foi utilizada. Os episódios de quedas antes e após a cirurgia foram estimados. Resultados O escore médio de Lequesne antes da cirurgia foi de 15,95 e após a cirurgia foi de 6,5. Esse resultado foi estatisticamente significativo $(p<0,001)$. 0 escore médio da EDG antes da cirurgia foi de 7,43 e após a ATJ foi de 2,22 $(p<0,001)$. 0 número médio de ocorrências de queda antes do procedimento, em um período de 1 ano, foi de 1,22 e após a ATJ foi de $0,27(p=0,004)$. Foi encontrada uma relação direta entre os escores de Lequesne antes da cirurgia e os escores da $\operatorname{EDG}(p=0,004)$.

Conclusões A ATJ resultou em melhora da dor e limitação funcional, diminuição ou desaparecimento da condição depressiva e diminuição das taxas de queda nos pacientes avaliados.

Objective To assess the preoperative and postoperative outcomes of patients diagnosed with severe knee osteoarthritis who underwent a total knee arthroplasty (TKA) using a mobile-bearing implant with a rotating platform and removing the posterior cruciate ligament. The present study focused on the outcomes relative to depression, pain, functional limitations, and fall episodes.
Trabalho desenvolvido na Faculdade de Medicina, Universidade Unichristus, Fortaleza, CE, Brasil. recebido

30 de Setembro de 2019 aceito

20 de Dezembro de 2019
DOI https://doi.org/

10.1055/s-0040-1708519. ISSN 0102-3616.
Copyright $\odot 2020$ by Sociedade Brasileira License terms de Ortopedia e Traumatologia. Published by Thieme Revinter Publicações Ltda, Rio de Janeiro, Brazil 


\author{
Keywords \\ - arthroplasty, \\ replacement, knee \\ - osteoarthritis \\ - depression
}

Methods The Lequesne questionnaire was used to assess pain and functional limitations before and after TKA. In addition, the geriatric depression scale (GDS) was also used. Episodes of falls before and after the surgery were estimated.

Results The mean Lequesne score before the surgery was 15.95 , and that after surgery was 6.5. This finding was statistically significant $(p<0.001)$. The mean GDS score before the surgery was 7.43, and that after TKA was $2.22(p<0.001)$. The mean number of fall occurrences before the procedure, over a 1-year period, was 1.22 , and that after TKA was $0.27(p=0.004)$. A direct relationship was found between the Lequesne scores before the surgery and the GDS scores $(p=0.004)$.

Conclusions Total knee arthroplasty resulted in the improvement of pain and functional limitation, decrease or disappearance of the depressive condition, and decrease of fall rates in the evaluated patients.

\section{Introdução}

Existem fortes evidências mostrando uma associação entre osteoartrite grave e distúrbios psiquiátricos, como ansiedade e depressão. A depressão é mencionada em vários estudos como um distúrbio muito frequente geralmente causado por dor crônica, limitações funcionais, histórico de quedas e comprometimento das funções sociais. ${ }^{1,2}$

A artroplastia total do joelho (ATJ) tem sido cada vez mais utilizada em ortopedia. À medida que implantes anatômicos mais modernos e resistentes são desenvolvidos, melhores resultados são esperados., ${ }^{3,4} \mathrm{O}$ aumento da expectativa de vida da população mundial se correlaciona com uma maior incidência de doenças degenerativas. Portanto, o número de ATJs deverá aumentar nas próximas décadas. ${ }^{5,6}$ Nos Estados Unidos, espera-se um aumento de $69 \%$ na incidência desta cirurgia em 2050, em relação ao ano de $2012 .^{7}$

A osteoartrite (OA) progride com dor e limitações funcionais, prejudicando o desempenho das atividades diárias simples. Alguns estudos também mostram um aumento da incidência de depressão e quedas em pacientes com OA..$^{8-10}$

$\mathrm{O}$ objetivo do presente estudo foi avaliar as taxas de depressão, dor e limitações funcionais e a frequência de quedas em pacientes com OA antes e após a ATJ. A hipótese era que os pacientes submetidos à ATJ apresentariam menos dor, limitações funcionais, quedas e depressão após a cirurgia.

\section{Materiais e Métodos}

Este é um estudo retrospectivo que incluiu pacientes com diagnóstico de OA de joelho, com mais de 55 anos de idade, e dispostos a assinar o Termo de Consentimento Livre e Esclarecido (TCLE). Os critérios de inclusão foram ter um prontuário completo contendo informações que pudessem ser analisadas pelos pesquisadores e uma recomendação ortopédica para ATJ com prótese de joelho de plataforma rotativa após exame físico e de imagem. A indicação do tratamento cirúrgico foi baseada na gravidade da dor e nas limitações funcionais confirmadas pelo exame físico ou pela refratariedade ao tratamento clínico. Esses pacientes apre- sentavam OA grau 3 ou superior, de acordo com a classificação de Ahlbäck. Foram excluídos os pacientes com menos de 55 anos de idade, os que não desejavam assinar a TCLE, aqueles com prontuários incompletos, e pacientes sem diagnóstico de OA do joelho ou não indicados para cirurgia com prótese de plataforma rotativa.

Foram incluídos 37 pacientes com escore de Lequesne igual ou superior a 8 , e 32 pacientes foram excluídos. Todos os pacientes foram submetidos a tratamento e cirurgia em um hospital filantrópico terciário, no país do estudo, entre janeiro de 2015 e janeiro de 2016. Todos os 37 pacientes receberam uma prótese de plataforma rotativa no joelho, seguindo uma técnica baseada na teoria do alinhamento mecânico com equalização espacial.

Os dados foram coletados de janeiro de 2015 a janeiro de 2017 e arquivados em fevereiro de 2017. O questionário de Lequesne foi utilizado antes da cirurgia e 5 meses após o procedimento, juntamente com a escala de depressão geriátrica (EDG). Além disso, os pesquisadores foram treinados para fazer aos pacientes as mesmas perguntas sobre quedas antes e após a cirurgia. As quedas antes e após a cirurgia deviam estar relacionadas à dor, limitações funcionais, ou instabilidade do joelho, ocorrendo dentro de um ano antes ou após a cirurgia. Todos os pesquisadores foram treinados para aplicar os questionários de Lequesne e EDG para obter consistência. Os pesquisadores decidiram que ortopedistas e radiologistas não teriam acesso aos resultados da pontuação de Lequesne até que o estudo fosse concluído.

O questionário de Lequesne et al. ${ }^{11}$ foi introduzido em 1987 para avaliar a dor e as limitações funcionais e foi revisado por Faucher et al. ${ }^{12}$ em 2003. Esta ferramenta de avaliação consiste em um questionário autoaplicável contendo 11 perguntas sobre dor, desconforto e função. Dependendo da pontuação, o paciente é classificado como tendo uma deficiência leve (1-4 pontos), moderada (5-7 pontos), severa (8-10 pontos), muito severa (11-13 pontos), ou extremamente severa (maior ou igual a 14 pontos). A versão da EDG utilizada neste estudo teve 15 questões, nas quais uma pontuação com soma maior ou igual a 6 pontos indicava depressão. ${ }^{13}$ 
O software IBM SPSS Statistics para Windows, versão 20.0 (IBM Corp., Armonk, EUA) foi utilizado para a análise estatística. O teste da hipótese do Qui-quadrado foi utilizado para investigar a associação entre variáveis categóricas e distribuição da amostra. 0 teste de Wilcoxon foi utilizado para 0 número de quedas antes e depois da ATJ. Um valor de $p<0,05$ foi considerado estatisticamente significativo.

Este estudo seguiu princípios básicos de ética em pesquisa em seres humanos, como autonomia, justiça, beneficência e não maleficência, norteados pelaresolução 466/12 do Conselho Nacional de Saúde. O estudo foi submetido ao Comitê de Ética em Pesquisa em Seres Humanos (CEP), utilizando a plataforma do país de estudo, enviado ao Centro Universitário e aceito de acordo com o protocolo no. 44595315.1.0000.5049.

\section{Resultados}

Antes da cirurgia, 70,3\% dos pacientes foram classificados como portadores de deficiências extremamente graves, $21,6 \%$ muito graves, e 8,1\% graves. Após a cirurgia, 13,5\% foram classificados como portadores de deficiência extremamente grave, $2,7 \%$ muito grave, $21,6 \%$ grave, $13,5 \%$ moderada, e $48,6 \%$ leve, conforme descrito no questionário de Lequesne (- Fig. 1). A pontuação média antes da cirurgia foi de 15,95 e após a cirurgia foi de 6,5. A redução do escore em cada paciente após a cirurgia foi estatisticamente significativa $(p<0,001)$ ( - Fig. 1 ).

A análise desses escores permitiu a identificação de certas características da dor dos pacientes: 63,2\% dos pacientes apresentaram dor durante o sono noturno, mesmo sem movimento, 75\% apresentaram dor matinal de até 15 minutos, e $68,4 \%$ afirmaram ter dor quando começaram a andar, que piorou com a caminhada contínua.

Quanto à frequência de quedas, o número médio de ocorrências antes da cirurgia no período de um ano foi de 1,22. O número médio de quedas após ATJ no período de um ano foi de 0,27 (-Tabela 1). Foi observado um número
Tabela 1 Episódios de quedas e escala de depressão geriátrica

\begin{tabular}{|l|l|l|l|}
\hline & $\begin{array}{l}\text { Média antes } \\
\text { da cirurgia }\end{array}$ & $\begin{array}{l}\text { Média depois } \\
\text { da cirurgia }\end{array}$ & Valor de $p$ \\
\hline $\begin{array}{l}\text { Episódios } \\
\text { de quedas }\end{array}$ & 1,22 & 0,27 & $p=0,004$ \\
\hline EDG & 7,43 & 2,22 & $p<0,001$ \\
\hline
\end{tabular}

Abreviatura: Escala de depressão geriátrica.

reduzido de quedas após ATJ em 73,68\% dos pacientes $(p=0,004)$. O teste de Wilcoxon também foi aplicado a essa variável, com nível de significância de $p=0,003$.

Quanto à EDG, o escore médio antes da cirurgia foi de 7,43, diminuindo para 2,22 após a ATJ (- Tabela 1). Essa redução também foi estatisticamente significativa $(p<0,001)$. Além disso, quanto maior a pontuação de Lequesne de um paciente antes da cirurgia, maior a pontuação da $\operatorname{EDG}(p=0,004)$.

\section{Discussão}

O questionário Lequesne foi desenvolvido na França na década de 1970 e é amplamente utilizado na Europa para avaliar pacientes com $\mathrm{OA}$. Ele contém várias perguntas sobre dor, desconforto e função que devem ser respondidas pelos pacientes. A gravidade dos sintomas e as limitações da capacidade física podem ser avaliadas em pacientes com OA de qualquer país ou cultura. ${ }^{14}$

Quanto aos escores antes e após a cirurgia, um estudo francês relatou que o escore médio de seus pacientes antes e após a artroplastia foi de 14,5 e 7,9 , respectivamente. ${ }^{15}$ No presente estudo, o escore médio antes da ATJ foi maior, 15,95, e a média após a ATJ foi menor, 6,5 $(p<0,001)$.

As quedas podem ser comuns em pacientes com OA do joelho devido à dor, limitações funcionais e atrofia muscular causada pela doença. $\mathrm{O}$ número esperado de quedas deve ser

Lequesne antes e após a cirurgia

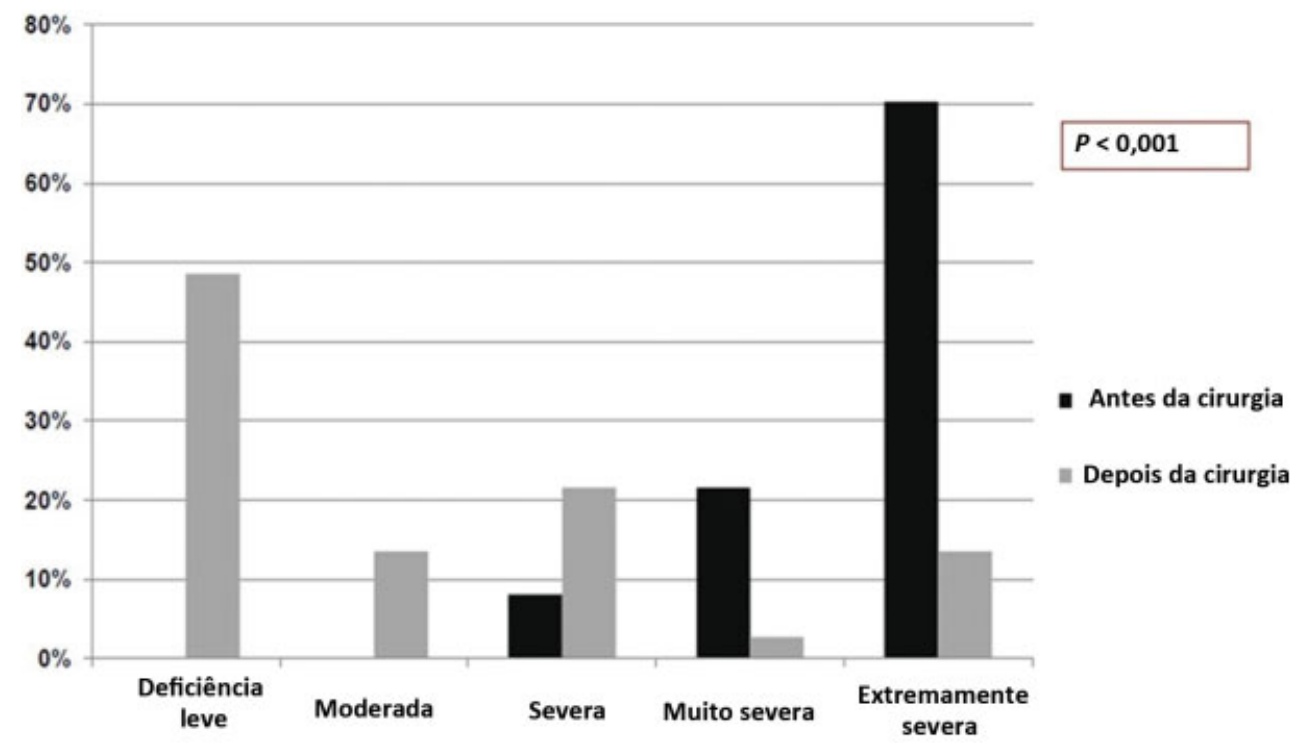

Fig. 1 Escores de Lequesne antes e após a cirurgia (fonte: autor). 
menor $^{16}$ após uma ATJ bem-sucedida, com uma diferença significativa nos escores de Lequesne antes e após a cirurgia. Neste estudo, o número de quedas após a cirurgia foi significativamente menor $(p<0,05)$.

Um total de $80 \%$ dos pacientes que sofriam quedas não caiu novamente após a ATJ, o que está muito próximo dos dados fornecidos por outro estudo que mostrou um total de $76 \%{ }^{16}$ Em um estudo britânico, os estudiosos apresentaram um total de $54,2 \%$ dos pacientes que sofriam quedas que não caíram novamente. ${ }^{8}$ Alguns pacientes mais velhos podem ter esquecimento e isso pode ser uma limitação para este estudo. As verificações de dados de queda semanais ou mensais provavelmente devem ser mais precisas.

Alguns estudos relataram alta prevalência de depressão em pacientes com OA. ${ }^{8,17,18}$ A EDG é amplamente utilizada para rastrear depressão em idosos. ${ }^{13}$ É considerada uma escala confiável e importante que também pode ser usada para estimar o risco de suicídio. ${ }^{19}$ A EDG foi utilizada no presente estudo para rastrear a depressão e avaliar se os sintomas depressivos melhoraram após a cirurgia. Dessa forma, assim como alguns estudos internacionais, este estudo mostrou uma diminuição estatisticamente significativa $(p<0,05)$ na depressão do paciente. ${ }^{17,18}$ Além disso, quanto maior a pontuação de Lequesne de um paciente antes da cirurgia, maior a pontuação da $\operatorname{EDG}(p=0,004)$. Não temos conhecimento de outros estudos correlacionando o escore de Lequesne ao questionário da EDG.

O número de pacientes selecionados para este estudo pode ser considerado uma limitação. Requisitos de inclusão, questionários com informações ausentes e pacientes que não responderam a um dos questionários reduziram o número de pacientes na amostra.

Devido à importância da depressão e suas consequências durante o tratamento e para a satisfação do paciente antes e após a cirurgia, são necessários mais estudos para verificar a importância e a eficiência de um acompanhamento psicológico e/ou psiquiátrico antes e após a ATJ.

\section{Conclusão}

A ATJ pode diminuir a dor, as limitações funcionais, o número de quedas e a depressão. Além disso, no presente estudo, observamos que quanto maior o escore de Lequesne de um paciente, maior o escore da EDG. Isso sugere a presença de uma associação entre dor e limitações funcionais e a ocorrência de transtorno depressivo.

Conflito de Interesses

Os autores declaram não haver conflito de interesses.

\section{Referências}

1 Vennu V, Bindawas SM. Relationship between falls, knee osteoarthritis, and health-related quality of life: data from the Osteoarthritis Initiative study. Clin Interv Aging 2014;9:793-800
2 Sheng J, Liu S, Wang Y, Cui R, Zhang X. The Link between Depression and Chronic Pain: Neural Mechanisms in the Brain. Neural Plast 2017;2017:9724371

3 Garcez-Leme LE, Sitta MC, Toledo Ma, Henriques SS. Cirurgia ortopédica em idosos: aspectos clínicos. Rev Bras Ortop 2011; 46(03):238-246

4 Liow MH, Chin PL, Tay KJ, Chia SL, Lo NN, Yeo SJ. Early experiences with robot-assisted total knee arthroplasty using the DigiMatch ${ }^{\mathrm{TM}}$ ROBODOC ${ }^{\circledR}$ surgical system. Singapore Med J 2014;55(10): 529-534

5 Piano LP, Golmia RP, Scheinberg M. Artroplastia total de quadril e joelho: aspectos clínicos na fase perioperatória. Einstein (Sao Paulo) 2010;8(3 Pt 1):350-353

6 Guenther D, Schmidl S, Klatte TO, et al. Overweight and obesity in hip and knee arthroplasty: Evaluation of 6078 cases. World J Orthop 2015;6(01):137-144

7 Inacio MCS, Paxton EW, Graves SE, Namba RS, Nemes S. Projected increase in total knee arthroplasty in the United States - an alternative projection model. Osteoarthritis Cartilage 2017;25 (11):1797-1803

8 Axford J, Butt A, Heron C, et al. Prevalence of anxiety and depression in osteoarthritis: use of the Hospital Anxiety and Depression Scale as a screening tool. Clin Rheumatol 2010;29 (11):1277-1283

9 Axford J, Heron C, Ross F, Victor CR. Management of knee osteoarthritis in primary care: pain and depression are the major obstacles. J Psychosom Res 2008;64(05):461-467

10 Parmelee PA, Harralson TL, McPherron JA, DeCoster J, Schumacher HR. Pain, disability, and depression in osteoarthritis: effects of race and sex. J Aging Health 2012;24(01):168-187

11 Lequesne MG, Mery C, Samson M, Gerard P. Indexes of severity for osteoarthritis of the hip and knee. Validation-value in comparison with other assessment tests. Scand J Rheumatol Suppl 1987; 65:85-89

12 Faucher M, Poiraudeau S, Lefevre-Colau MM, Rannou F, Fermanian J, Revel M. Assessment of the test-retest reliability and construct validity of a modified Lequesne index in knee osteoarthritis. Joint Bone Spine 2003;70(06):521-525

13 Sheikh JI, Yesavage JA. Geriatric depression scale (GDS): recent evidence and development of a shorter version. Clin Gerontol 1986;5(1/2):165-173

14 Lequesne MG. The algofunctional indices for hip and knee osteoarthritis. J Rheumatol 1997;24(04):779-781

15 Merle-Vincent F, Couris CM, Schott AM, et al. Osteoarthritis Section of the French Society for Rheumatology. Factors predicting patient satisfaction 2 years after total knee arthroplasty for osteoarthritis. Joint Bone Spine 2011;78(04):383-386

$16 \mathrm{Si} \mathrm{HB}$, Zeng Y, Zhong J, et al. The effect of primary total knee arthroplasty on the incidence of falls and balance-related functions in patients with osteoarthritis. Sci Rep 2017;7(01):16583

17 Bistolfi A, Bettoni E, Aprato A, et al. The presence and influence of mild depressive symptoms on post-operative pain perception following primary total knee arthroplasty. Knee Surg Sports Traumatol Arthrosc 2017;25(09):2792-2800

18 Duivenvoorden T, Vissers MM, Verhaar JA, et al. Anxiety and depressive symptoms before and after total hip and knee arthroplasty: a prospective multicentre study. Osteoarthritis Cartilage 2013;21(12):1834-1840

19 Heisel MJ, Flett GL, Duberstein PR, Lyness JM. Does the geriatric depression scale (GDS) distinguish between older adults with high versus low levels of suicidal ideation? Am J Geriatr Psychiatry 2005;13(10):876-883 